\title{
Biparametric MRI of the prostate
}

\author{
Michele Scialpi ${ }^{1}$, Alfredo D’Andrea ${ }^{2}$, Eugenio Martorana ${ }^{3}$, Corrado Maria Malaspina ${ }^{1}$, Maria Cristina Aisa ${ }^{4}$, \\ Maria Napoletano ${ }^{1}$, Emanuele Orlandi ${ }^{1}$, Valeria Rondoni ${ }^{1}$, Pietro Scialpi ${ }^{5}$, Diamante Pacchiarini ${ }^{6}$, Diego Palladino \\ Michele Dragone ${ }^{7}$, Giancarlo Di Renzo ${ }^{4,8}$, Annalisa Simeone ${ }^{7}$, Giampaolo Bianchi ${ }^{9}$, Luca Brunese ${ }^{10}$
}

Cite this article as: Scialpi M, D’Andrea A, Martorana E, Malaspina C, Aisa MC, Napoletano M, et al. Biparametric MRI of the prostate. Turk J Urol 2017; 43(4): 401-9

\begin{abstract}
${ }^{1}$ Department of Surgical and Biomedical Sciences, Division of Radiology 2, Santa Maria della Misericordia Hospital, Perugia University, Sant' Andrea delle Fratte, Perugia, Italy

${ }^{2}$ Department of Experimenta Medicine, Magrassi Lanzara, Luigi Vanvitelli, Second University of Naples, Naples, Italy

${ }^{3}$ Department of Urology, Clinica Castelli, Bergamo, Italy

${ }^{4}$ Department of Surgical and Biomedical Sciences, Division of Gynaecology, Santa Maria della Misericordia Hospital, Perugia University, Sant' Andrea delle Fratte, Perugia, Italy

${ }^{5}$ Division of Urology, Portogruaro Hospital, Venice, Italy

${ }^{6}$ Health Management, S. Maria della Misericordia Hospital, Sant' Andrea delle Fratte, Perugia, Italy

${ }^{7}$ Department of Radiology, Casa Sollievo della Sofferenza Hospital, Foggia, Italy

${ }^{8} 3$ DIFIC, Medical Area, University of Perugia, Perugia, Italy

${ }^{9}$ Division of Urology, University of Modena, Modena, Italy

${ }^{10}$ Department of Radiology, Campobasso University, C.da
\end{abstract} Tappino, Campobasso, Italy

Submitted:

13.10.2017

Accepted:

27.10.2017

Correspondence:

Michele Scialpi

E-mail:

michelescialpi1@gmail.com

(C) Copyright 2017 by Turkish

Association of Urology

Available online at

www.turkishjournalofurology.com

\begin{abstract}
Biparametric Magnetic Resonance Imaging (bpMRI) of the prostate combining both morphologic T2weighted imaging (T2WI) and diffusion-weighted imaging (DWI) is emerging as an alternative to multiparametric MRI (mpMRI) to detect, to localize and to guide prostatic targeted biopsy in patients with suspicious prostate cancer (PCa). BpMRI overcomes some limitations of mpMRI such as the costs, the time required to perform the study, the use of gadolinium-based contrast agents and the lack of a guidance for management of score 3 lesions equivocal for significant PCa. In our experience the optimal and similar clinical results of the bPMRI in comparison to mpMRI are essentially related to the DWI that we consider the dominant sequence for detection suspicious PCa both in transition and in peripheral zone. In clinical practice, the adoption of bpMRI standardized scoring system, indicating the likelihood to diagnose a clinically significant PCa and establishing the management of each suspicious category (from 1 to 4), could represent the rationale to simplify and to improve the current interpretation of mpMRI based on Prostate Imaging and Reporting Archiving Data System version 2 (PI-RADS v2). In this review article we report and describe the current knowledge about bpMRI in the detection of suspicious PCa and a simplified PI-RADS based on bpMRI for management of each suspicious PCa categories to facilitate the communication between radiologists and urologists.
\end{abstract}

Keywords: Biparametric magnetic resonance imaging; prostate cancer; prostate imaging reporting and data system.

\section{Introduction}

Prostate cancer $(\mathrm{PCa})$ is the most common cancer among men and the second and third leading cause of cancer-related death in the United States and Europe, respectively. ${ }^{[1-3]}$ Accurate detection and localization of the suspicious PCa is of paramount importance for appropriate management. Diagnostic algorithm based on several parameters [digital rectal examination, serum prostate-specific antigen (PSA) and transrectal ultrasound (TRUS)-guided prostate biopsy and nomograms] provides limited information for this purpose. ${ }^{[4]}$

Magnetic resonance imaging (MRI) of the prostate with T2-weighted imaging (T2WI) was first described in the mid-1980s..$^{[5,6]} \mathrm{T} 2 \mathrm{WI}$ suffered from relatively poor sensitivity and specificity for detecting $\mathrm{PCa}$ and showed moderate accuracy in loco-regional staging. ${ }^{[7]}$ T2WI and dynamic contrast-enhanced (DCE) MRI protocol with endorectal coil (ERC) have not improved the diagnostic performance of MRI in the detection of PCa especially in the transition zone (TZ). A sensitivity of $55-88 \%$ and $46-90 \%$ and a specificity of $67-82 \%$ and 74-96\% were reported by different authors for T2WI and DCE-MRI, respectively. ${ }^{[8-10]}$ The introduction of diffusion-weighted imaging (DWI) by measurement of the restriction of water diffusion of suspicious lesions has significantly improved the detection and localization of PCa in clinical practice. ${ }^{[11-13]}$ 
State-of-the-art high field strength (1.5T and 3T) MRI technologies in multiparametric MRI (mpMRI) of the prostate (incorporating T2WI, DWI and DCE sequences) represents the most comprehensive diagnostic approach for $\mathrm{PCa}$ and it is actually considered the reference standard imaging modality in detecting, localizing and staging prostate cancer. ${ }^{[14-17]}$ MpMRI interpretation is based on Prostate Imaging and Reporting Archiving Data System version 2 (PI-RADS v2) ${ }^{[18]}$, introduced to improve standardization of MRI interpretation by reporting suspicion scores (1- to 5-point scale based on fixed criteria) to provide clinical management guideline for suspicious $\mathrm{PCa}$.

MpMRI demonstrated that $\mathrm{PCa}$ is often multifocal and increased the sensitivity of conventional MRI in the detection of $\mathrm{PCa}$, especially involving $\mathrm{TZ}$ and anterior fibromuscular stroma. ${ }^{[19,20]}$ MpMRI has some limitations such as its higher cost, longer time required to complete the study and the use of gadolinium-based contrast agents. Moreover, PI-RADS v2 does not offer a clear guidance for clinical management of PI-RADS score 3 lesions. ${ }^{[18]}$ Biparametric MRI (bpMRI), including T2W and DW MRI series, has been recently proposed to overcome these limitations and to offer a more appropriate management of $\mathrm{PCa} \cdot{ }^{[21-28]}$

The benefits of bpMRI are represented by the reduced costs, shorter time required to complete the study, and the no-use of gadolinium. In addition, bpMRI provides similar results to those of mpMRI for the detection and localization of PCa. ${ }^{[24,29]}$

Different studies have also demonstrated the validity of the lesion volume as predictor of PCa and aggressiveness of the lesion. ${ }^{[30]}$ Several authors have also reported a good correlation between radiologic estimation of the lesion volume and histology of the tumor volüme. ${ }^{[19,25,30]}$

A potential of bpMRI is represented by the management (biopsy or clinical surveillance) of the score 3 lesions through the calculation of their volume (cut-off of $0.5 \mathrm{~cm}^{3}$ ) which is consequently a simplification of PI-RADS v2. In this article we review the bpMRI of the prostate and its performance in the detection, localization and management of $\mathrm{PCa}$.

\section{Clinical history}

The following information should be available before MRI acquisition and interpretation in men with suspicious PCa: PSA values (recent, and previous), family history for $\mathrm{PCa}$, persistently, and highly suspicious prostatic lesion despite negative standard 12-core biopsy, previous diagnosis of High Grade Prostatic Intraepithelial Neoplasia (HGPIN) or Atypical Small Acinar Proliferation (ASAP).

\section{Biparametric MRI implementation and technical requirements}

A diagnostic prostate bpMRI is achievable at $1.5 \mathrm{~T}$ field strength, even when not using ERC or preferentially by $3 \mathrm{~T}$ system if available. In our 6 years experience, bpMRI has been performed using 3T scanner (Achieva, Philips Medical Systems, Healthcare, Eindhoven, the Netherlands) with 16-channels torso phasedpelvic array. Imaging with high signal to noise ratio (SNR) of the entire gland, capsule and neurovascular bundles is obtained, and lesions have been accurately detected, and localized. Patients with contraindications for MRI have to be excluded. When possible, patients should evacuate the rectum just prior to the MRI exam. Administration of antispasmolytics such as butylscopolamine may be helpful to reduce intestinal peristalsis.

\section{T2-weighted imaging}

High-resolution T2WI turbo-spin echo (TSE) allows morphological informations depicting prostate gland anatomy [peripheral zone (PZ), TZ, capsule and neurovascular bundle] and any suspicious lesions. The T2WI-TSE sequence is acquired in the axial, sagittal and coronal planes. It allows measurement of both prostate and lesion volume, localizes suspicious $\mathrm{PCa}$ within the gland and guides targeted biopsy. In addition, sagittal and coronal planes are useful for evaluating extracapsular extension (ECE) of PCa (e.g. seminal vesicle invasion).

On T2WI, PCa localized in PZ appears predominantly and distinctly hypointense compared to the hyperintense glandular tissue of the $\mathrm{PZ}^{[31]}$, and when ECE is recognizable on T2WI, it must be interpreted as a reliable sign of malignancy. In TZ different but morphologically similar entities (e.g. post-inflammatory or post-biopsy scars, atrophic changes, prostatitis, granulomatous prostatitis, intraepithelial neoplasia (PIN) or post-treatment lesions and often benign prostatic hyperplasia $[\mathrm{BPH}])^{[32-34]}$ that appear as focal hypointense areas, may mimic PCa.

Post-biopsy hemorrhages (generally persisting for several months after biopsy, so it is suggested an interval of at least six weeks before performing MRI) ${ }^{[18]}$ also appear hypointense on T2WI but hyperintense on fat-suppressed T1-weighted gradient-echo sequence with High-Resolution Isotropic Volume Excitation (THRIVE). In addition, an axial TSE T2W high resolution spectral pre-saturation inversion recovery (HR SPIR) sequence, using an extended field-of-view (FOV), allows the detection of enlarged parailiac and locoregional lymph nodes and/or skeletal metastases.

PI-RADS v2 considers T2WI the dominant sequence in the detection of PCa localized in TZ. T2WI is performed using an echo time (TE) of 100-120 ms and a long repetition time (TR) of $4000-8000 \mathrm{~ms}$ (depending on the equipment and B0 
field strength), a minimum slice thickness of $3 \mathrm{~mm}$ at $1.5 \mathrm{~T}$ or at 3T with no gap, and a minimum in-plane resolution of $0.7 \times$ $0.7 \mathrm{~mm}$ for both field strengths. In practice we perform T2WI acquisitions on axial, coronal and sagittal planes to detect more rapidly the lesions and to asses extraglandular extension of disease. On our 3T system a FOV approximately of $18 \mathrm{~cm}$, with matrices of $212 \times 212$ (frequency $\times$ phase) and slice thicknesses of $2 \mathrm{~mm}$ with no gap is used.

\section{Diffusion-Weighted Imaging}

DWI analyzes the movement (diffusion) of water molecules and expresses it by a parameter known as the apparent diffusion coefficient (ADC). Restriction of water diffusion in PCa, due to numerous intact cell membranes and scarce extracellular spaces, with a resultant decrease in the ADC value, is measured. PCa shows lower ADC or restricted diffusion surrounding healthy prostate tissue and appears hyperintense on the diffusionweighted (DWI) images with high b-value and hypointense on ADC maps ${ }^{[35,36]}$ The different numbers and magnitudes of the selected $b$-values may determine variations in the ADC results related to different field strengths. ${ }^{[37]}$

Although several studies showed the potential of quantitative ADC values for differentiating high Gleason score (GS) from low GS PCa, an overlapping between benign and malignant lesions exists and ADC values for this differentiation vary among different MR systems. PI-RADS $\mathrm{v} 2^{[18]}$ considers DWI the dominant sequence to identify tumors in $\mathrm{PZ}$, while in our experience we have considered DWI the dominant sequence for detection of PCa in both $\mathrm{TZ}$ and $\mathrm{PZ}$ and only visual analysis of lesions on ADC map has been performed.

DWI is generally performed with a single-shot echo-planar (SS-EPI) sequence in the same axial orientation of the T2WI. Diffusion gradients should be applied in 3 orthogonal spatial directions. A minimum of 3, ideally 5, b-values $>1000 \mathrm{~s} / \mathrm{mm}^{2}$ should be used. TE should be as short as possible (typically $<90$ $\mathrm{ms})$. The sequence is prone to susceptibility artefacts that may lead to distortions of the DW images due to adjacent bowel gas.
The measurement of restricted diffusion in tumor tissue using high b-values improves diagnostic accuracy of MRI for PCa.

Minimum slice thickness of $3 \mathrm{~mm}$ with no gap and 2 number of excitations (NEX) should be used. B-values $0-2000 \mathrm{~s} / \mathrm{mm}^{2}$ with ADC map are generated. According to PI-RADS v2, a maximum b-value of $2000 \mathrm{~s} / \mathrm{mm}^{2}$ may be used. DWI with very high b-values may be challenging in some systems due to excessive loss of SNR, particularly if imaging at $1.5 \mathrm{~T}$ or without using ERC is obtained..$^{[38]}$

On our 3T system we perform DWI acquisitions on axial plane, slice thicknesses of $2 \mathrm{~mm}$ with no gap using $124 \times 100$ (frequency x phase) matrices, a FOV of $28-32 \mathrm{~cm}$, and 4 b-values 0-2000 $\mathrm{s} / \mathrm{mm}^{2}$ with reconstruction of ADC maps. BpMRI acquisition protocol and sequence parameters are presented in Table 1.

\section{Biparametric vs multiparametric MRI in the detection of prostate cancer}

PI-RADS v2 and several studies ${ }^{[18,20,27,28,39]}$ recognized that the data supporting the role of DCE are limited and the added value provided by DCE to T2WI and DWI appears to be modest. Costs, time required to perform the study, gadolinium-based contrast media potential risk in patients with impaired renal function and its accumulation in the central nervous system represent the major drawbacks of DCE. ${ }^{[40]}$

The value of bpMRI in the detection of PCa using 1.5T MRI with endorectal coil has been reported. ${ }^{[23,41]}$ Recently, it has been demonstrated that the sensitivity of bpMRI at $3 \mathrm{~T}$ without endorectal coil in the detection PCa is similar to that of mpMRI. $[20,24,27,28,42-44]$

The diagnostic value of bpMRI in men with or without prior biopsy and combined with PSA has been validated, resulting in an improved accuracy for detecting clinically significant PCa (sPCa) and representing an effective technique to direct biopsy needles under TRUS guidance, after MRI-ultrasonography fusion. ${ }^{[20,23,28]}$

Table 1. Biparametric MIRI of the prostate at 3T: acquisition protocol and sequence parameters

\begin{tabular}{|c|c|c|c|c|c|c|c|}
\hline & Sequences & ST (mm) & Gap & TR (ms) & TE (ms) & FOV (8 mm) & Voxel size \\
\hline DWI & 2D-EPI & 2 & 0 & 3700 & 67 & $280 \times 320$ & $1.46 \times 1.45 \times 2$ \\
\hline T2W Axial & TSE & 2 & 0 & 12700 & 90 & $180 \times 180$ & $0.28 \times 0.28 \times 2$ \\
\hline T2W Coronal & TSE & 2 & 0 & 8400 & 90 & $180 \times 180$ & $0.28 \times 0.28 \times 2$ \\
\hline T2W Sagitta & TSE & 2 & 0 & 8400 & 90 & $180 \times 180$ & $0.28 \times 0.28 \times 2$ \\
\hline T1W Axial & THRIVE & 1.5 & 0 & 3.0 & 1.43 & $300 \times 370$ & $1.46 \times 1.46 \times 1.5$ \\
\hline
\end{tabular}

DWI: diffusion-weighted imaging; EPI: echo planar imaging; TSE: turbo spin echo; THRIVE: Tl High-Resolution Isotropic Volume Excitation; ST: slice thickness; TR: time of repetition; TE: time of echo; FOV: field of view 
Table 2. Diagnostic accuracy and sensitivity of biparametric and multiparametric MRI in the detection prostate cancer at $3 \mathrm{~T}$ without endorectal coil: literature review

\begin{tabular}{lccccc}
\hline Authors & Year & bpMRI accuracy & mpMRI accuracy & bpMRI sensitivity & mpMRI sensitivity \\
\hline Radtke et al. ${ }^{[20] *}$ & 2015 & - & - & $91.9 \%$ & $86.4-88.5 \%$ \\
\hline Fascelli et al. ${ }^{[28]}$ & 2016 & $81.4 \% * *$ & - & $95.5 \%$ & - \\
\hline Thestrup et al. ${ }^{[42]}$ & 2016 & $41.5 \%$ & $39 \%$ & $94.6 \%$ & $93-100 \%$ \\
\hline Stanzione et al. ${ }^{[44]}$ & 2016 & $92.7 \%$ & $93.9 \%$ & $83.5 \%$ & $91.1 \%$ \\
\hline Scialpi et al. ${ }^{[24]} * * *$ & $99.4 \%$ & $99.4 \%$ & $98.2 \%$ & $98.2 \%$ \\
\hline De Visschere et al. ${ }^{[27]}$ & 2017 & $72.2-74.7 \%$ & $72.9 \%$ & $61.8-72.2 \%$ & $72.9 \%$ \\
Kuhl et al. ${ }^{[43]}$ & 2017 & $89.1 \%$ & $87.2 \%$ & $93.9 \%$ & $84.6 \%$ \\
\hline *Anterior lesions GS $\geq 3+4$ and GS $\geq 4+3 ; * *$ Overall accuracy (bpMRI and PSA); ***index lesions $\geq 10 \mathrm{~mm}$. & & \\
\hline
\end{tabular}

For diagnosis of clinically sPCa in patients with elevated PSA before biopsy, DCE was found to be an unnecessary imaging technique in the majority of patients evaluated by PI-RADS v2. ${ }^{[27,42]}$ In our experience, the high sensitivity of DWI/ADC associated with T2WI, corresponding to the performance of DWI alone $(97.6 \%, 100 \%$ and $94.7 \%$ for, respectively, all lesions, $\mathrm{PZ}$ and $\mathrm{TZ}$ ), was equal to that of mpMRI for lesions in $\mathrm{PZ}, \mathrm{TZ}$ and in the anterior fibromuscular stroma. ${ }^{[24]}$ The sensitivity rates of bpMRI and mpMRI at 3T without ERC in the detection of PCa are 61.8-97.6\%, and 72.9-97.6\%, respectively (Table 2). ${ }^{[20,24,27,28,42-44]}$

\section{Biparametric MRI: lesion detection and localization}

According to the criteria and lexicon of the PI-RADS v2 guidelines ${ }^{[18]}$, the bpMRI images' analysis is based on the recognition of lesion patterns on T2WI and DWI and corresponding ADC maps. Because in our experience DWI represents the predominant sequence to detect lesions both in $\mathrm{PZ}$ and $\mathrm{TZ}$ with confirmed image quality, for routine clinical work we recommend starting viewing DWI with high b-values (and the same sequence inverted) on ADC maps and subsequently at T2WI.

The index lesion is detected as a focal area with hyperintensity on DWI with high b-values and mild/moderate or marked hypointensity on ADC maps, and moderate or marked hypointensity on T2WI. A standard bpMRI layout of our prostate image display is shown in Figure 1.

Prostate is analyzed from the seminal vesicles to the apex for the detection of focal lesions localized in the PZ and TZ. Findings based on bpMRI are described and at maximum 4 index lesions are identified and localized according to the 39 sector/region- prostate map model reported in PI-RADS v $2^{[18]}$ within approximately 15 minutes. In cases with significant enlargement of $\mathrm{TZ}$ and presence of median lobe, to localize lesions in this side, we propose a 41 sector/region- prostate map model, by adding two segments (right and left) of an intravescical median lobe (Figure 2).

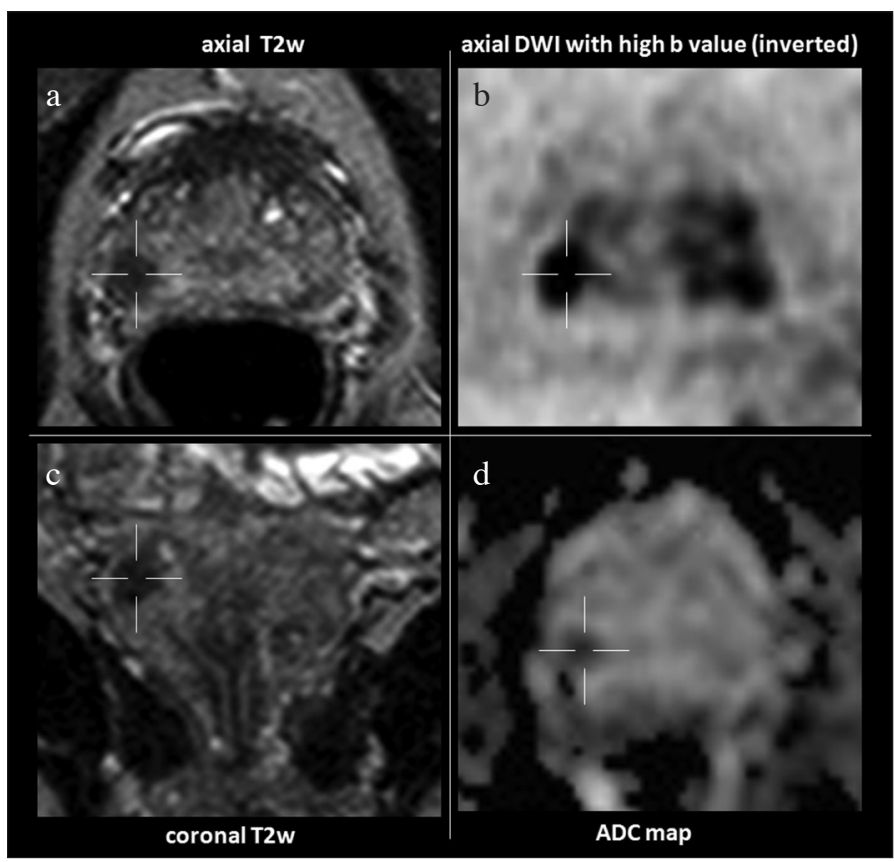

Figure 1. a-d. Standard layout of prostate image display in a one screen setting. Double clicking in one of the image allows to display this image in full screen mode. On the left side the morphologic axial and sagittal T2W images and on the right side DWI with high b-values (inverted) and ADC map are displayed. Ideally the morphologic $\mathrm{T} 2 \mathrm{~W}$ and the DW images should be linked at the same table position. With this described display mode, detection and localization of the suspected lesions (hypointense on $\mathrm{T} 2 \mathrm{~W}$, hyperintense on DWI with high b-values or hypointense on inverted with corresponding decrease on ADC map), can already be carried out.

\section{Biparametric MRI volume calculation for the management of score 3 lesions}

One of the major drawback of PI-RADS v2 is that it does not offer a precise guidance on clinical management (biopsy or clinical surveillance) of score 3 lesions (equivocal for clinical sPCa). 


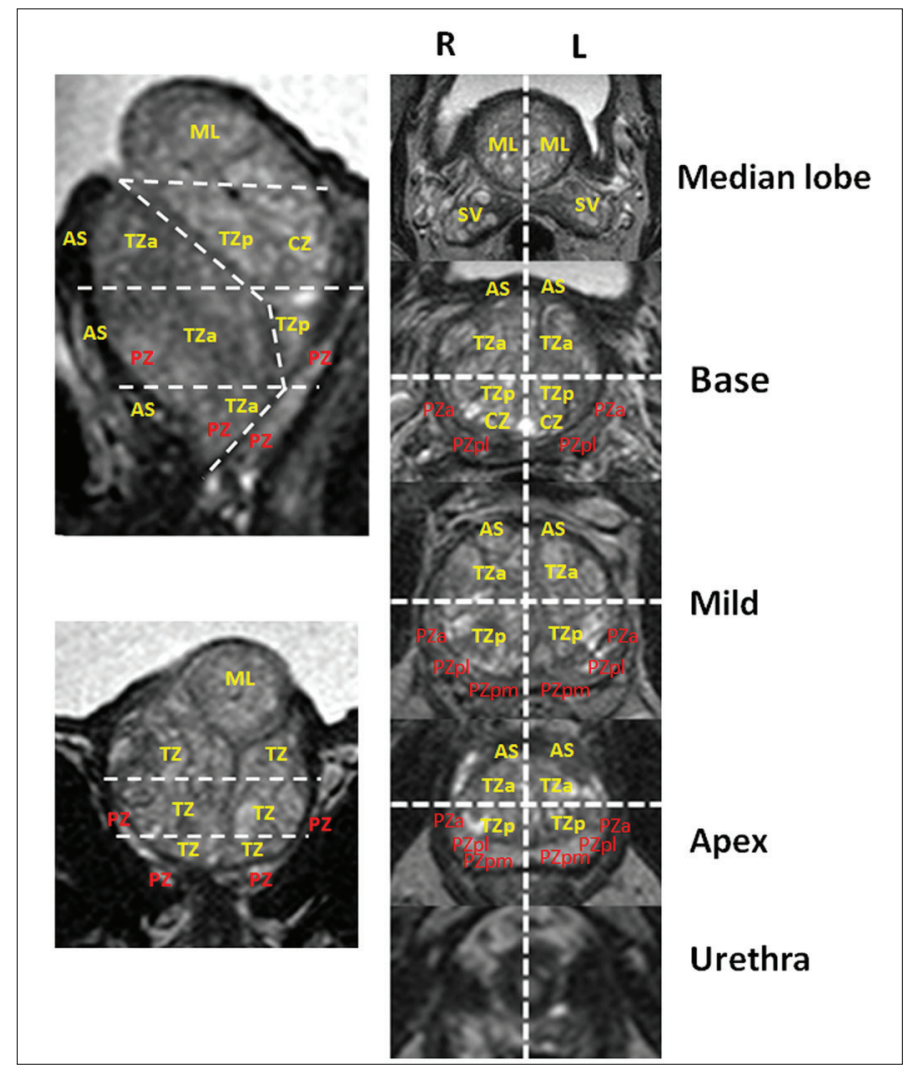

Figure 2. The 41 segmentation model proposed for prostate with enlargement of transition zone and presence of median intravescical lobe: fourthy-one sectors/regions: thirty-eight for the prostate, two for the seminal vesicles and one for the external urethral sphincter are identified. In comparison to 39 segmentation model proposed by PI-RADS v2, the 41 segmentation model adds two segments (right and left) of an intravescical median lobe.

TZ: transizon zone; CZ: central zone; PZ: peripheral zone; AS: anterior stroma; SV: seminal vesicle; ML: median lobe.

Some authors biopsize all PI-RADS score 3 lesions ${ }^{[44]}$ leading to increased number of unnecessary biopsies, on the other hand, others have demonstrated that PI-RADS score 3 lesions are associated with low likelihood of clinically sPCa, concluding that these lesions should not be sampled but monitored only. ${ }^{[45]}$ Several studies ${ }^{[25,29]}$ have demonstrated the adequacy of MRI lesion volume estimation compared to histopathological tumor volume. According to the Epstein criteria ${ }^{[46]}$, the presence of sPCa should be predicted by lesion volume measurement, using a cut-off value of $0.5 \mathrm{~cm}^{3}$.

We discriminated two subgroups as $3 \mathrm{a}\left(<0.5 \mathrm{~cm}^{3}\right)$ and $3 \mathrm{~b}(>0.5$ $\mathrm{cm}^{3}$ ) PI-RADS v2 3 score lesions (lesions both in PZ and in TZ, moderately hypointense on $\mathrm{T} 2$, hyperintense on high b-value DWI and mildly/moderately hypointense on ADC map) ${ }^{[47]}$, proposed a simplified PI-RADS (S-PI-RADS) score based on
bpMRI and focused on the clinical management of PI-RADS v2 score 3 lesions. S-PI-RADS scoring system consists of 4 lesion categories (from 1 to 4 ), considers volumes of all score 3 lesions and includes all PI-RADS v2 score 4 and 5 lesions within a single risk category (score 4 ).

Lesion volume for score 3 lesions should be calculated using the ellipsoidal formula (width $\mathrm{x}$ height $\mathrm{x}$ length $\mathrm{x}$ 0.52) or alternatively using a software (Mimics Innovation Suite, Materialise NV, Leuven, Belgium) with three-dimensional (3D) reconstruction, drawing a freehand region of interest (ROI) around the discernible index lesion on the inverted DWI with a high b-value (Figure 3).

\section{S-PI-RADS: communication of findings and management} In our experience, the adoption of S-PI-RADS based on bpMRI of suspicious lesions offers the advantage of an easier communication of findings between radiologists and urologists and a precise indication for the management of each risk category. The S-PI-RADS scheme adapted to bpMRI and clinical management of each category is presented in Figure 4.

S-PI-RADS category 1 corresponds to PI-RADS v2 score 1 lesion: moderately homogeneous signal intensity (normal) on T2WI and no abnormality on DWI/ADC. Follow-up with PSA is indicated.

S-PI-RADS category 2 corresponds to PI-RADS v2 score 2 lesion: circumscribed hypointense area on T2WI without restriction on diffusion on DWI/ADC. Follow-up with PSA and bpMRI eventually within 2 years is indicated.

S-PI-RADS category 3 includes PI-RADS v2 score 3 lesion: focal rounded, lenticular or irregular lesion moderate heterogeneous or homogeneous hypointense on T2WI, mild/moderate hyperintense on DWI with high b-values and marked hypointense on ADC map. For lesions with volume $<0.5 \mathrm{~cm}^{3}$ (subgroup a) accurate evaluation of age and clinical information, periodic monitoring of PSA value and repetition of bpMRI 1 year later is suggested. This approach is related to the fact that indolent PCa remains stable over time from diagnosis ${ }^{[48]}$ and lesions with volume $<0.5 \mathrm{~cm}^{3}$ include a minimal percentage of GS score 7 adenocarcinoma (about $2 \%$ ). ${ }^{[30]} \mathrm{A}$ score 3 lesion becomes suitable of bioptic check if its volume reaches $0.5 \mathrm{~cm}^{3}$ during the follow-up, shifting from category 3 to 4 . For lesions with volume $>0.5 \mathrm{~cm}^{3}$ targeted biopsy is indicated.

S-PI-RADS category 4 includes PI-RADS v2 score 4 and 5 lesions. Heterogeneous or homogeneous mild/moderate or markedly hypointense focal rounded, lenticular or irregular lesions on T2WI, hyperintense on high b-value DWI and marked hypoin- 


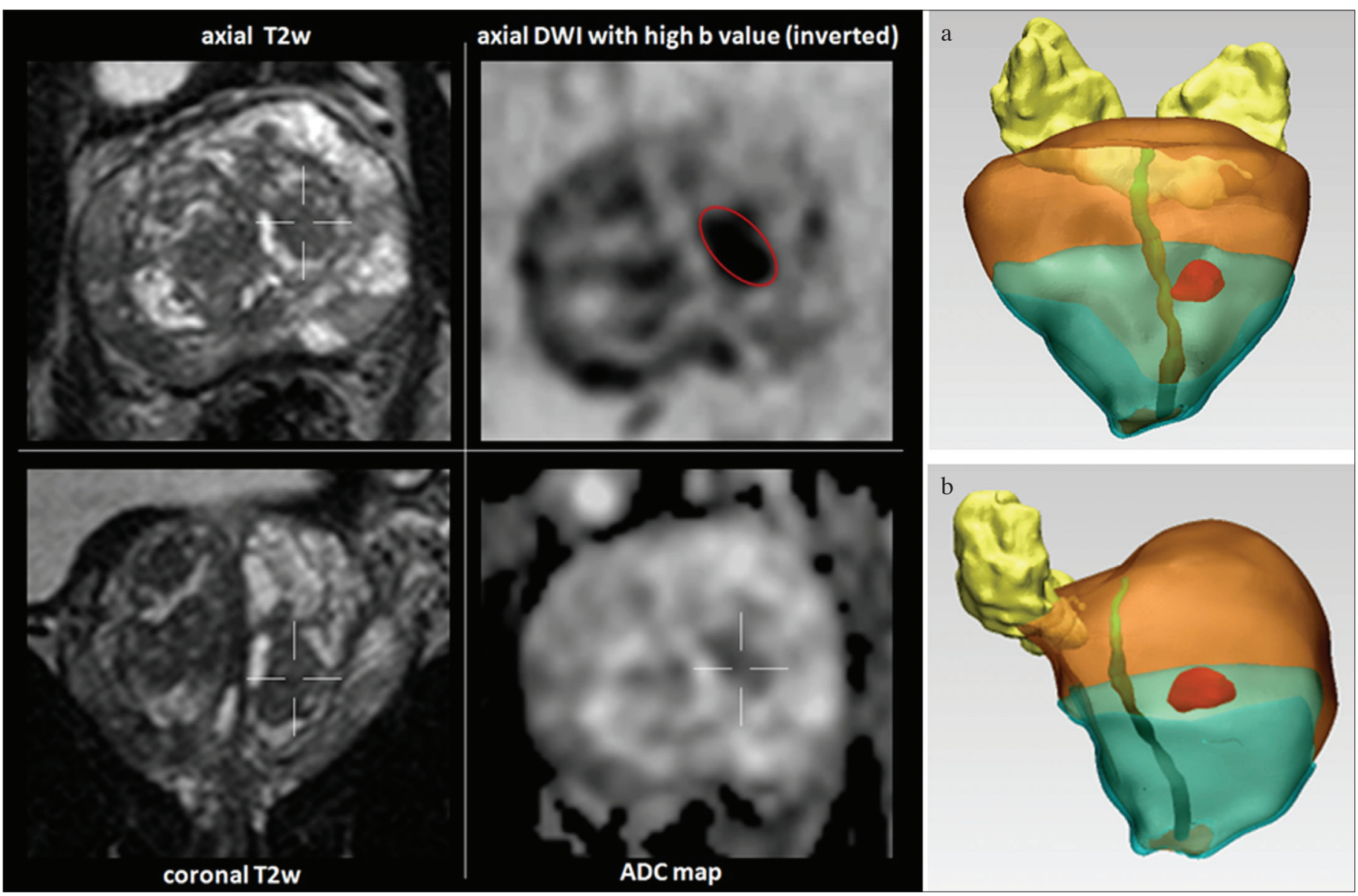

Figure 3. a, b. Lesion score 3 lesions detected in the transizion zone at mild of the prostate on the left side on morphologic axial and coronal T2W images and DWI with high b-values and ADC map. Three-dimensional (3D) reconstruction coronal (a) and oblique (b) is obtained by drawing a freehand region of interest (ROI) around the discernible index lesion on the inverted DWI with high b-value

tense on ADC map. PI-RADS v2 score 4 are intraglandular lesions and PI-RADS v2 score 5 are lesions extending outside the prostate or bulging the capsule and/or invading the seminal vesicles. Targeted biopsy is indicated.

Thereafter, the entire pelvis should be analyzed for lymph node involvement, bone metastases and other findings. As reported, especially DW sequences of the entire pelvis in combination with meticulous analysis of T2W STIR sequence help to detect lymph node metastases. ${ }^{[49]}$

At our institution we formulate the report in a structured way on a free text basis. The bpMRI report includes clinical notes, technical details, findings [prostate volume, detection and localization of the lesion (at maximum 4 lesions are indicated), volume calculation for score 3 lesions, assessment of the lesion's category and its management]. Besides possible ECE and infiltration of the neurovascular bundle and seminal vesicles as well as infiltration of adja- cent organs, exact location, number and size of possible lymph node metastases in the entire pelvis, and bone metastases are also recorded.

The knowledge of already categorized and reported pitfalls (normal anatomic structures, noncancerous abnormalities and technical challenges related to DW sequences) in $\mathrm{PCa},{ }^{[50]}$ may aid the radiologist to reduce false-positive results obtained with bpMRI. These pitfalls, of which the radiologist have to be aware of, are presented in Table 3 .

\section{Conclusion}

BpMRI (morphological T2WI and DWI) allows an accurate detection and localization of suspicious PCa, in addition to reduction of the time required to complete the study, lower costs without using gadolinium. BpMRI and lesion volume measurement for PI-RADS score 3 lesions discriminate 4 categories of lesions for each of which a corresponding 


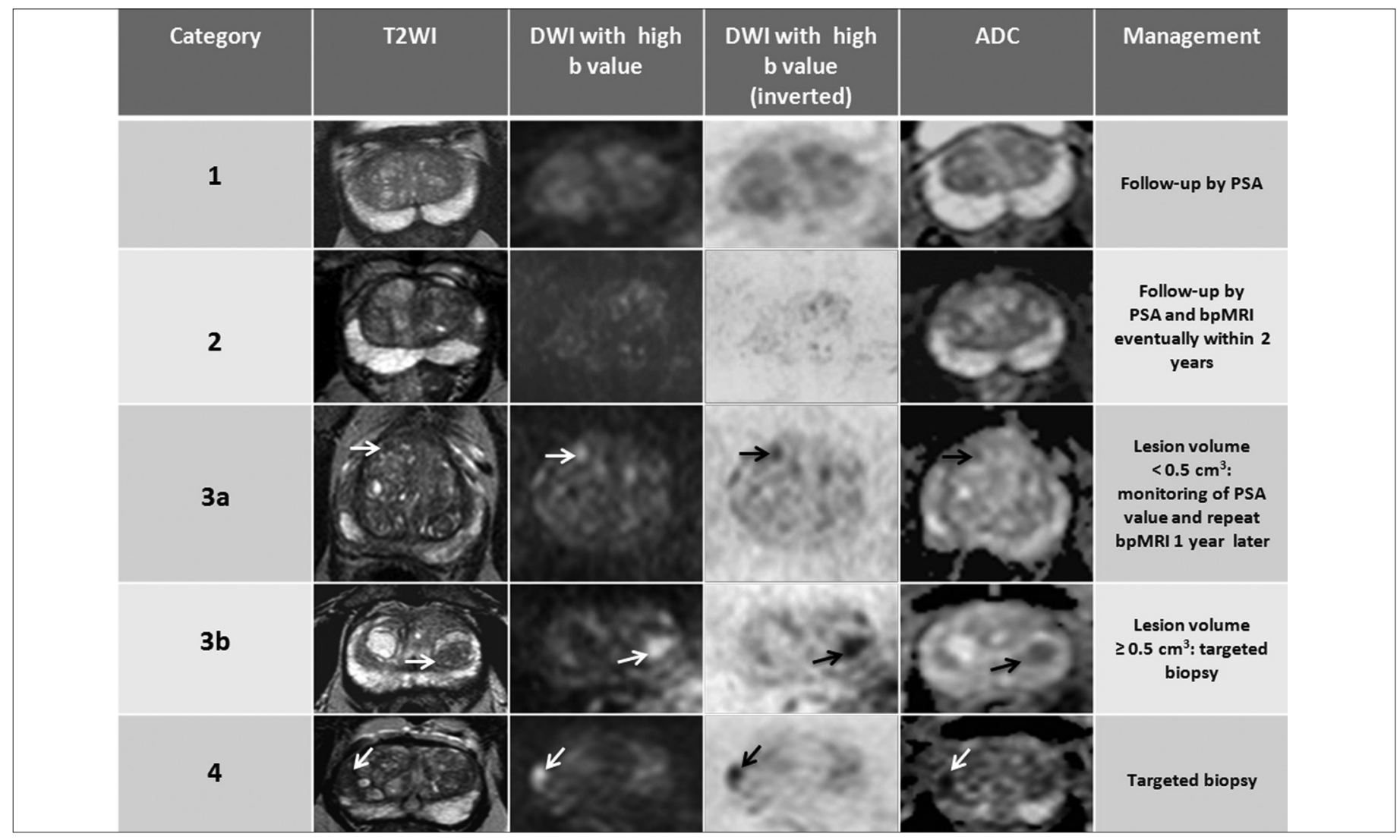

Figure 4. Simplified PI-RADS (S-PI-RADS) based on biparametric MRI

S-PI-RADS category 1: homogeneous intermediate signal intensity (normal) on T2WI and no anormality on DWI/ADC. Follow-up by PSA is indicated.

S-PI-RADS category 2: focal rounded, lenticular or irregular mild/moderately or markedly hypointensity area on T2WI without restriction on diffusion on DWI/ADC. Follow-up by PSA and biparametric MRI eventually within 2 years is indicated.

S-PI-RADS category 3: includes 1): subgroup a (lesion volume: $<0.5 \mathrm{~cm} 3$ : round lesion moderately hypointense on T2WI (arrow), hyperintense on high b-value DWI (arrow) and inverted(arrow), and moderately hypointense on ADC map (arrow): accurate evaluation of age, clinical informations, periodic monitoring of PSA value and repetition of bpMRI 1 year later is suggested; 2) subgroup b (lesion volume: $>0.5 \mathrm{~cm} 3$ ): lenticular lesion moderately hypointense on T2WI (arrow), hyperintense on high b-value DWI (arrow) and inverted (arrow), and moderately hypointense on ADC map (arrow). Targeted biopsy is indicated.

S-PI-RADS category 4: includes PI-RADS v2 score 4 (intraglandular lesions) and 5 (lesions extending to an area outside of the prostate or bulging the capsule of the prostate and/or invasion of the seminal vesicles lesions). Focal lenticular lesion markedly hypointense on T2WI (arrow), hyperintense on high b-value DWI (arrow) and inverted (arrow), and markedly hypointense on ADC map (arrow). Targeted biopsy is indicated.

\section{Table 3. Pitfalls that can mimic prostate cancer on biparametric MRI}

Normal anatomic structures

Noncancerous abnormalities

Technical challenges related to DWI
Normal central zone or periprostatic venous plexus or neurovascular bundle can mimic peripheral zone lesions. Bilateral T2w image hypointensities at the base and/or median posterior T2w image hypointense area in the middle third of the gland can mimic peripheral zone lesions. Asymmetric thickening of the surgical capsule can be difficult to distinguish from a focal lesion.

Post-biopsy haemorrhage.

Differentiation between stromal benign prostatic hyperplasia and a transition zone tumour. Benign prostatic hyperplasia (BPH) nodule protrusion (can mimic a peripheral zone lesion). Acute and chronic prostatitis, post-inflammatory scars, granulomatous prostatitis and atrophy.

Anatomic distortion or lack of suppression of benign prostate tissue on standard high b-value DWI can lead to false positive findings. Suboptimal windowing of ADC maps can determine false negative results. 
decision-making is indicated. In clinical practice the adoption of S-PI-RADS based on bpMRI represents a potential valid system that facilitates lesion management and communication with other professional colleagues.

Peer-review: This manuscript was prepared by the invitation of the Editorial Board and its scientific evaluation was carried out by the Editorial Board.

Author Contributions: Concept - M.S., A.D.A., E.M., C.M.M., A.S., G.B., L.B.; Design - M.S., A.D.A., E.M., C.M.M.; Supervision - M.S., A.D.A., E.M., C.M.M., A.S., D.P.; Data Collection and/or Processing - M.S., A.D.A., E.M., C.M.M., A.S., D.P., M.D.; Analysis and/or Interpretation - M.S., A.D.A., E.M., C.M.M.; Literature Search - M.S., C.M.M., M.N., E.O., V.R., M.C.A., D.P.; Writing Manuscript - M.S., A.D.A., E.M., C.M.M., L.B.; Critical Review - M.S., A.D.A., E.M., C.M.M., P.S., M.N., V.R., G.D.R., M.C.A., D.P., G.B., L.B.

Acknowledgements: The Authors thank for 3DIFIC the Mr Marco Pergentini for his contribute in 3D reconstruction, and the technologist Domenico Mezzasoma for his assistance in the evaluation of MRI sequence parameters.

Conflict of Interest: No conflict of interest was declared by the authors.

Financial Disclosure: The authors declared that this study has received no financial support.

\section{References}

1. Siegel R, Naishadham D, Jemal A. Cancer statistics, 2013. CA Cancer J Clin 2013;63:11-30. [Crossref]

2. Jemal A, Center MM, DeSantis C, Ward EM. Global patterns of cancer incidence and mortality rates and trends. Cancer Epidemiol Biomarkers Prev 2010;19:1893-907. [Crossref]

3. Ferlay J, Autier P, Boniol M, Heanue M, Colombet M, Boyle P, et al. Estimates of the cancer incidence and mortality in Europe in 2006. Ann Oncol 2007;18:581-92. [Crossref]

4. Fine SW, Amin MB, Berney DM, Bjartell A, Egevad L, Epstein JI, et al. A contemporary update on pathology reporting for prostate cancer: biopsy and radical prostatectomy specimens. Eur Urol 2012;62:20-39. [Crossref]

5. Poon PY, McCallum RW, Henkelman MM, Bronskill MJ, Sutcliffe $\mathrm{SB}$, Jewett MA, et al. Magnetic resonance imaging of the prostate. Radiology 1985;154:143-9. [Crossref]

6. Hricak H, Dooms GC, Jeffrey RB, Avallone A, Jacobs D, Benton WK, et al. Prostatic carcinoma: staging byclinical assessment, CT, and MR imaging. Radiology 1987;162:331-6. [Crossref]

7. Tempany CM, Rahmouni AD, Epstein JI, Walsh PC, Zerhouni EA. Invasion of the neurovascular bundle by prostate cancer: evaluation with MR imaging. Radiology 1991;181:107-12. [Crossref]

8. Yoo S, Kim JK, Jeong IG. Multiparametric magnetic resonance imaging for prostate cancer: A review and update for urologists. Korean J Urol 2015;56:487-97. [Crossref]
9. Wu LM, Xu JR, Ye YQ, Lu Q, Hu JN. The clinical value of diffusion-weighted imaging in combination with T2-weighted imaging in diagnosing prostate carcinoma: a systematic review and metaanalysis. AJR Am J Roentgenol 2012;199:103-10. [Crossref]

10. Verma S, Turkbey B, Muradyan N, Rajesh A, Cornud F, Haider MA, et al. Overview of dynamic contrast-enhanced MRI in prostate cancer diagnosis and management. AJR Am J Roentgenol 2012;198:1277-88. [Crossref]

11. Bammer R. Basic principles of diffusion-weighted imaging. Eur J Radiol 2003;45:169-84. [Crossref]

12. Tan CH, Wang J, Kundra V. Diffusion weighted imaging in prostate cancer. Eur Radiol 2011;21:593-603. [Crossref]

13. Haider MA, van der Kwast TH, Tanguay J, Evans AJ, Hashmi AT, Lockwood G, et al. Combined T2-weighted and diffusion-weighted MRI for localization of prostate cancer. AJR Am J Roentgenol 2007;189:323-8. [Crossref]

14. Rais-Bahrami S, Siddiqui MM, Turkbey B, Stamatakis L, Logan $\mathrm{J}$, Hoang AN, et al. Utility of multiparametric magnetic resonance imaging suspicion levels for detecting prostate cancer. J Urol 2013;190:1721-8. [Crossref]

15. Siddiqui MM, Rais-Bahrami S, Truong H, Stamatakis L, Vourganti $\mathrm{S}$, Nix J, et al. Magnetic resonance imaging/ultrasound-fusion biopsy sig nificantly upgrades prostate cancer versus systematic 12-core transrectal ultrasound biopsy. Eur Urol 2013;64:713-9. [Crossref]

16. Bloch BN, Genega EM, Costa DN, edrosa I, Smith MP, Kressel HY, et al. Prediction of prostate cancer extracapsular extension with high spatial resolution dynamic contrast-enhanced 3-T MRI. Eur Radiol 2012;22:2201-10. [Crossref]

17. de Rooij M, Hamoen EH, Fütterer JJ, Barentsz JO, Rovers MM. Accuracy of multiparametric MRI for prostate cancer detection: a meta-analysis. AJR Am J Roentgenol 2014;202:343-51. [Crossref]

18. Prostate Imaging Reporting and Data System (PI-RADS) Reston (VA): American College of Radiology. Available at:http://www. acr.org/Quality-Safety/ Resources/PIRADS/ Accessed March 5, 2015.

19. Radtke JP, Schwab C, Wolf MB, Freitag MT, Alt CD, Kesch C, et al. Multiparametric Magnetic Resonance Imaging (MRI) and MRI-Transrectal Ultrasound Fusion Biopsy for Index Tumor Detection: Correlation with Radical Prostatectomy Specimen. Eur Urol 2016;70:846-53. [Crossref]

20. Radtke JP, Boxler S, Kuru TH, Wolf MB, Alt CD, Popeneciu IV, et al. Improved detection of anterior fibromuscular stroma and transition zone prostate cancer using biparametric and multiparametric MRI with MRI-targeted biopsy and MRI-US fusion guidance. Prostate Cancer Prostatic Dis 2015;18:288-96. [Crossref]

21. Scialpi M, Falcone G, Scialpi P, D'Andrea A. Biparametric MRI: a further improvement to PIRADS 2.0? Diagn Interv Radiol 2016;22:297-8. [Crossref]

22. Scialpi M, Martorana E, D’Andrea A. Standardizing biparametric MRI to simplify and improve Prostate Imaging Reporting and Data System, version 2, in prostate cancer management. AJR Am J Roentgenol 2016;6:W1-2. [Crossref]

23. Rais-Bahrami S, Siddiqui MM, Vourganti S, Turkbey B, Rastinehad AR, Stamatakis L, et al. Diagnostic value of biparametric mag- 
netic resonance imaging (MRI) as an adjunct to prostate-specific antigen (PSA)-based detection of prostate cancer in men without prior biopsies. BJU Int 2015;115:381-8. [Crossref]

24. Scialpi M, Prosperi E, D’Andrea A, Martorana E, Malaspina C, Palumbo B, et al. Biparametric versus Multiparametric MRI with Non-endorectal Coil at 3T in the Detection and Localization of Prostate Cancer. Anticancer Res 2017;37:1263-71. [Crossref]

25. Baco E, Ukimura O, Rud E, Vlatkovic L, Svindland A, Aron M, et al. Magnetic resonance imaging-transectal ultrasound imagefusion biopsies accurately characterize the index tumor: correlation with step-sectioned radical prostatectomy specimens in 135 patients. Eur Urol 2015;67:787-94. [Crossref]

26. Radtke JP, Schwab C, Wolf MB, reitag MT, Alt CD, Kesch C, et al. Multiparametric magnetic resonance imaging (MRI) and MRItransrectal ultrasound fusion biopsy for index tumor detection: correlation. Eur Urol 2016;70:846-53. [Crossref]

27. De Visschere P, Lumen N, Ost P, Decaestecker K, Pattyn E, Villeirs G. Dynamic contrast-enhanced imaging has limited added value over T2-weighted imaging and diffusion-weighted imaging when using PI-RADSv2 for diagnosis of clinically significant prostate cancer in patients with elevated PSA. Clin Radiol 2017;72:23-32. [Crossref]

28. Fascelli M, Rais-Bahrami S, Sankineni S, Brown AM, George AK, Ho R, et al. Combined biparametric prostate magnetic resonance imaging and prostate-specific antigen in the detection of prostate cancer: a validation study in a biopsy-naive patient population. Urology 2016;88:125-34. [Crossref]

29. Scialpi M, Rondoni V, Aisa MC, Martorana E, D’Andrea A, Malaspina CM, et al. Is contrast enhancement needed for diagnostic prostate MRI? Transl Androl Urol 2017;6:499-509. [Crossref]

30. Martorana E, Pirola GM, Scialpi M, Micali S, Iseppi A, Bonetti LR, et al. Lesion volume predicts prostate cancer risk and aggressiveness: validation of its value alone and matched with prostate imaging reporting and data system score. BJU Int 2017;120:92103. [Crossref]

31. Hricak H. Imaging prostate cancer. J Urol 1999;162:1329-30. [Crossref]

32. Barentsz JO, Richenberg J, Clements R, Choyke P, Verma S, Villeirs G, et al. ESUR prostate MR guidelines 2012. Eur Radiol 2012;22:746-57. [Crossref]

33. Oto A, Kayhan A, Jiang Y, Tretiakova M, Yang C, Antic T, et al. Prostate cancer: differentiation of central gland cancer from benign prostatic hyperplasia by using diffusion-weighted and dynamic contrast-enhanced MR imaging. Radiology 2010;257:715-23. [Crossref]

34. Kim CK, Park BK, Kim B. Localization of prostate cancer using 3T MRI: comparison of T2-weighted and dynamic contrast-enhanced imaging. J Comput Assist Tomogr 2006;30:7-11. [Crossref]

35. Zelhof B, Pickles M, Liney G, Gibbs P, Rodrigues G, Kraus $\mathrm{S}$, et al. Correlation of diffusion-weighted magnetic resonance data with cellularity in prostate cancer. BJU Int 2009;103:883-8. [Crossref]

36. Sato C, Naganawa S, Nakamura $T$, Kumada $H$, Miura $S$, Takizawa O, et al. Differentiation of noncancerous tissue and cancer lesions by apparent diffusion coefficient values in transi- tion and peripheral zones of the prostate. J Magn Reson Imaging 2005;21:258-62. [Crossref]

37. Mueller-Lisse UG, Mueller-Lisse UL, Zamecnik P, Schlemmer HP, Scherr MK. Diffusion-weighted MRI of the prostate. Radiologe 2011;51:205-14. [Crossref]

38. Wang X, Qian Y, Liu B, Cao L, Fan Y, Zhang JJ, et al. High-bvalue diffusion-weighted MRI for the detection of prostate cancer at 3 T. Clin Radiol 2014;69:1165-70. [Crossref]

39. Barentsz JO, Weinreb JC, Verma S, Thoeny HC, Tempany CM, Shtern F, et al. Synopsis of the PI-RADS v2 Guidelines for Multiparametric Prostate Magnetic Resonance Imaging and Recommendations for Use. Eur Urol 2016;69:41-9. [Crossref]

40. Olchowy C, Cebulski K, Łasecki M, Chaber R, Olchowy A, Kałwak K, et al. The presence of the gadolinium-based contrast agent depositions in the brain and symptoms of gadolinium neurotoxicity - A systematic review. PLoS One 2017;12:e0171704. [Crossref]

41. Delongchamps NB, Rouanne M, Flam T, euvon F, Liberatore M, Zerbib M, et al. Multiparametric magnetic resonance imaging for the detection and localization of prostate cancer: combination of T2-weighted, dynamic contrast-enhanced and diffusion-weighted imaging. BJU Int 2011;107:1411-8. [Crossref]

42. Thestrup KC, Logager V, Baslev I, Møller JM, Hansen RH, Thomsen HS. Biparametric versus multiparametric MRI in the diagnosis of prostate cancer. Acta Radiol Open 2016;5:20-5. [Crossref]

43. Kuhl CK, Bruhn R, Krämer N, Nebelung S, Heidenreich A, Schrading S. Abbreviated Biparametric Prostate MR Imaging in Men with Elevated Prostate-specific Antigen. Radiology 2017;285:493-505. [Crossref]

44. Stanzione A, Imbriacoa M, Cocozza S, Fusco F, Rusconi G, Nappi C, Biparametric 3T Magentic Resonance Imaging for prostatic cancer detection in a biopsy-naïve patient population: a further improvement of PI-RADS v2? Eur J Radiol 2016;85:2269-74. [Crossref]

45. Liddell H, Jyoti R, Haxhimolla HZ. mp-MRI Prostate Characterised PIRADS 3 Lesions are Associated with a Low Risk of Clinically Significant Prostate Cancer - A Retrospective Review of 92 Biopsied PIRADS 3 Lesions. Curr Urol 2015;8:96-100. [Crossref]

46. Epstein JI, Walsh PC, Carmichael M, Brendler CB. Pathologic and clinical findings to predict tumor extent of nonpalpable (stage T1c) prostate cancer. JAMA 1994;271:368-74. [Crossref]

47. Scialpi M, Martorana E, Aisa MC, Rondoni V, D’Andrea A, Bianchi G. Score 3 prostate lesions: a gray zone for PI-RADS v2. Turk J Urol 2017;43:237-40. [Crossref]

48. Albertsen PC, Hanley JA, Fine J. 20-year outcomes following conservative management of clinically localized prostate cancer. JAMA 2005;293:2095-101. [Crossref]

49. Thoeny HC, Froehlich JM, Triantafyllou M, Huesler J, Bains LJ, Vermathen $\mathrm{P}$, et al. Metastases in Normal-sized Pelvic Lymph Nodes: Detection with Diffusion-weighted MR Imaging. Radiology 2014;273:125-35. [Crossref]

50. Rosenkrantz AB, Taneja SS. Radiologist, be aware: ten pitfalls that confound the interpretation of multiparametric prostate MRI. Am J Roentgenol 2014;202:109-20. [Crossref] 